\title{
Gummy Smile Treatment by Aesthetic Crown Lengthening on Altered Passive Eruption Case
}

\author{
Noer Ulfah, DDS, MDS and I Komang Evan Wijaksana, DDS \\ Department of Periodontology, Faculty of Dental Medicine, Universitas Airlangga, Surabaya, Indonesia
}

\begin{abstract}
An attractive smile enhances the appearance and acceptance of an individual in society. Gum exposure more than $3 \mathrm{~mm}$ is generally considered unattractive and known as a gummy smile, which is usually considered an aesthetic problem. At present, patients have a greater desire for more aesthetic results that may influence the planning of dental treatments. This case report aimed to describe the surgical sequence of aesthetic crown lengthening to improve smile profile and eliminate gummy smile. We reported a 21-year-old non-smoking woman with no pertinent medical history who presented with a chief complaint of an unattractive smile due to excessive gingival display. The gingival display in the smile was $5 \mathrm{~mm}$, and the width to height ratio of the central incisor was $121 \%$. Neither periodontal problems nor teeth mobility was detected. Assessment for the condition was excessive gingival display due to altered passive eruption. The overall prognosis for this case was good. The primary treatment plan proposed to the patient was an aesthetic crown lengthening. Altered passive eruption class I subtype A was a case conclusion, and aesthetic crown lengthening with gingivectomy without osseous reduction was the selected treatment. In conclusion, aesthetic crown lengthening should be considered as a surgical component of aesthetic therapy to improve smile profile and eliminate gummy smile.
\end{abstract}

Key Words: crown lengthening, gingivectomy, gummy smile

\section{INTRODUCTION}

An attractive smile increases the appearance and acceptance of individuals in society. The gingival tissue around the teeth plays an eminent role in the aesthetics of the anterior maxillary area. At present, patients have a greater desire for more aesthetic results that can affect dental care planning. Aesthetic considerations are very relevant respecting the relationship between the smile and facial beauty. ${ }^{1,2}$

The American Academy of Periodontology (AAP) defines gummy smile as a deformity and mucogingival condition that affects the area around the teeth. Gingiva that appears when smiling less than $2-3 \mathrm{~mm}$ can be considered attractive, whereas overexposure $(>3 \mathrm{~mm})$ is generally considered unattractive and is known as a gummy smile, which is usually considered an aesthetic problem. However,

Paper presented in the Temu Ilmiah Nasional-Internasional ke-8 (TIMNASS 8) on February 27-29, 2020 at Surabaya, Indonesia.

Corresponding author: Noer Ulfah, DDS, MDS

Department of Periodontology

Faculty of Dental Medicine

Universitas Airlangga

Surabaya, Indonesia

Email: noer-u@fkg.unair.ac.id / noer.u.fkgunair@gmail.com the perception of excessive gingival appearance also depends on cultural and ethnic preferences. ${ }^{3,4}$

Clinical crown lengthening is a periodontal resection procedure that aims to remove part of the supporting periodontal tissue to increase the exposure to the coronal tooth structure. Crown lengthening surgery has been categorized as aesthetic or functional. Aesthetic crown lengthening is usually performed in the aesthetic zone as 
in the upper maxillary anterior region with the purpose to reduce the appearance of the gingiva and increase the height of the clinical crown to improve the height and width of the tooth ratio. Central incisors are the dominant component in anterior teeth with an ideal width to height ratio of $75-80 \%$. The gingival margin for the lateral incisors must be $1 \mathrm{~mm}$ coronal to the central incisor, and the canine must be as high as the central incisor. On the other hand, functional crown lengthening is performed to expose the crown structure so that the functional prosthesis can be placed above the open crown. ${ }^{3,5}$

Therefore, the purpose of this case report is to describe the sequence of surgical aesthetic crown lengthening to increase the smiling profile and eliminate gummy smile.

\section{CASE REPORT}

A 21-year-old woman, a non-smoker, with no pertinent medical history visited Dental Hospital of Universitas Airlangga with a chief complaint of unattractive smile due to excessive gingival display. The patient has good oral hygiene and highly cooperates. At clinical examination, the keratinized gingiva band was 7 to $9 \mathrm{~mm}$ in width, and periodontal probing depth was $3 \mathrm{~mm}$ or less. The gingival display in the smile was $5 \mathrm{~mm}$. Neither periodontal problems nor teeth mobility was detected. Cast examination showed width to height ratio of the central incisor was $121 \%$. No radiographic abnormalities at the radiographic examination were detected. The periodontal ligament was within the normal limit, and the crown-to-root ratio was about 1:3. The assessment for the condition was excessive gingival display due to altered passive eruption. The overall prognosis for this case was good. The primary treatment plan proposed to the patient was the aesthetic crown lengthening of elements $13,12,11,21,22$, and 23 . The patient was informed about the treatment, and written consent was obtained following the local regulation.

\section{CASE MANAGEMENT}

Initially, a composite surgical guide was made before performing bleeding points. Composite surgical guide edge tangent to the cervical region as confirmed in T-bar tip of the proportional gauge for proportional width to height tooth ratio (Figure 1). The T-bar tip of the proportional gauge (Chu's Aesthetic gauges, $\mathrm{Hu}$-Friedy Inc, Chicago, IL) was used as the guide to establishing a correct dimension of clinical crowns. The gauge was placed in the center of the tooth, and it was observed that the red marks of the horizontal arm determined the width of the tooth; therefore, the red mark of the vertical arm indicated the height of the clinical crown.

Local anesthesia was administered with $2 \%$ mepivacaine (Scandonest 2\% special, Septodont, France) and bleeding points were performed to mark the desired height of the clinical crowns. The bone sounding was then performed
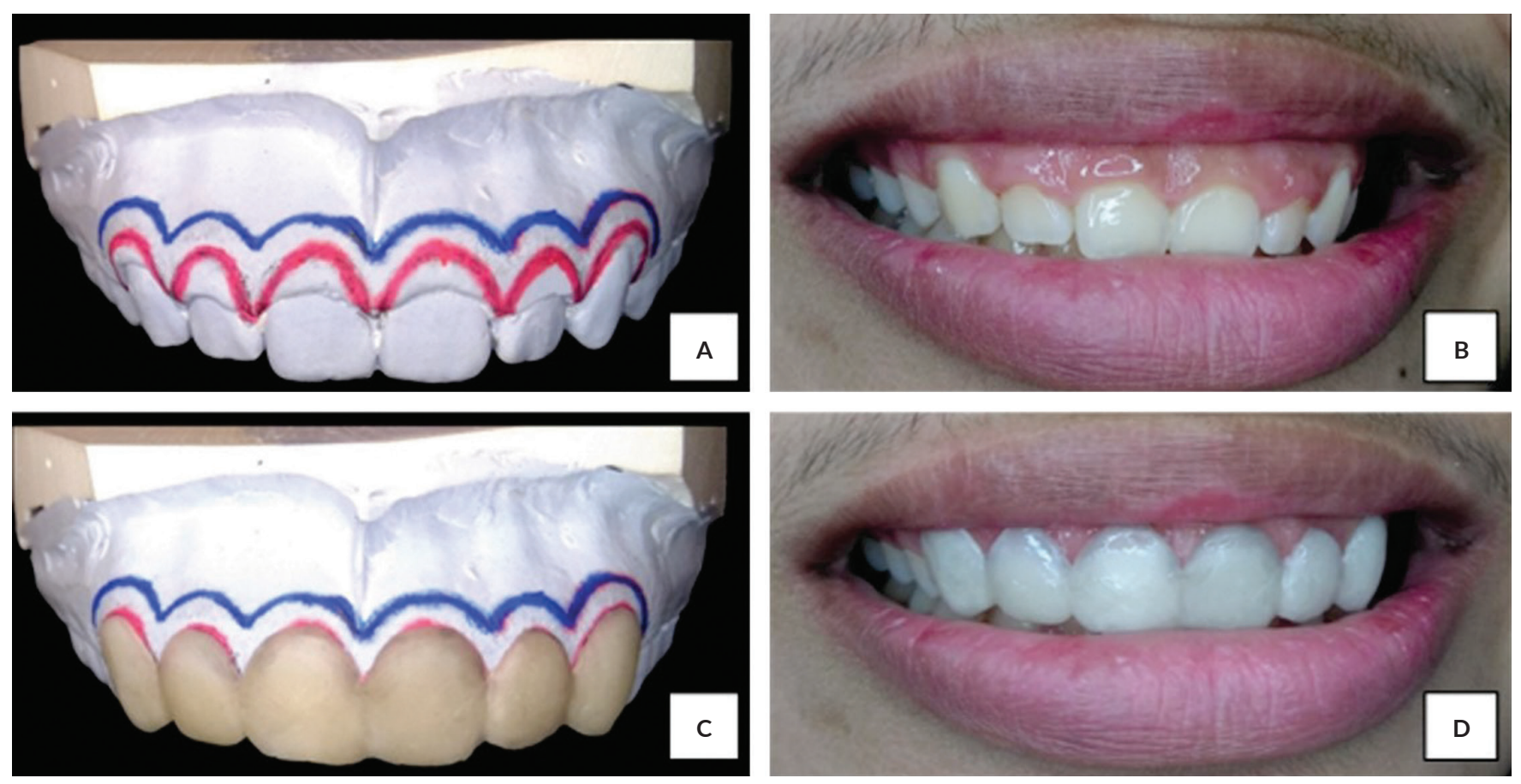

Figure 1. The initial condition of the patient. (A) The initial cast model, (B) The red line is the ideal gingival margin position for proportional width to length ratio. The blue line is the ideal position line for the alveolar crest. Initial clinical condition, (C) Composite mock-up in the cast, (D) Try in composite mock-up in the patient and smile analysis. 
with a bone sounding gauge (Hu-Friedy Inc, Chicago, IL) to measure the gingival margin to alveolar crest width, then deciding to perform gingival excision with or without osseous reduction (Figure 2A). In this case, the keratinized gingiva width was measured from $8-9 \mathrm{~mm}$, then the bone sounding 5-6 mm, thus excising $2-3 \mathrm{~mm}$ of gingiva will not make biological width violation. Thus, altered passive eruption class I subtype A was a case conclusion and aesthetic crown lengthening with gingivectomy without osseous reduction was the choice of treatment.

A gingivectomy procedure was then performed. Initial horizontal discontinues incision $1 \mathrm{~mm}$ apical to the bleeding point was made by using blade no.15C (Figure 2B). Secondary sulcular incision by using blade no.15C, followed by elimination of the excision gingiva with sickle then performed. Gingivoplasty was performed after all of six anterior teeth were proportional in width to height ratio, confirming T-bar tip of proportional gauge (Chu's Aesthetic gauges, Hu-Friedy Inc, Chicago, IL) (Figure 2CD). Periodontal dressing (Coe pack, GC Asia) was then applied at the site for about one week. Amoxicillin $500 \mathrm{mg}$ and mefenamic acid $500 \mathrm{mg}$ were prescribed three times a day for five days.

The first control, one week after the surgical phase, showed redness in the site area, but no pain experienced by the patient (Figure 3A). The patient was instructed to use chlorhexidine oral gargle twice a day for two weeks. The second control, two weeks after the surgical phase, showed a good result on the gingival with minimal redness and no pain. After one month, no clinical sign of inflammation was shown, and the patient was satisfied with her new smile profile (Figure 3C-D). The new proportion of width to length ratio was measured after one month. All the proportions are considered as esthetic proportional with width to length ratio of the anterior tooth around $75-80 \%$ (Table 1).

\section{DISCUSSION}

An altered passive eruption (APE) described as a condition where the relationship between the teeth, alveolar bone, and soft tissue creates an exaggerated appearance of the gingiva and can produce a "gummy smile." The study by Rossi et al. ${ }^{6}$ showed that $65 \%$ of patients with APE have a family history of the condition. many treatments have been introduced for the management of APE cases. ${ }^{6}$

As this case is classified as altered passive eruption class I subtype A, the case conclusion and aesthetic crown lengthening with gingivectomy without osseous reduction was the treatment of choice. Type I means that the gingival margin is located incisal or occlusal to the cementoenamel
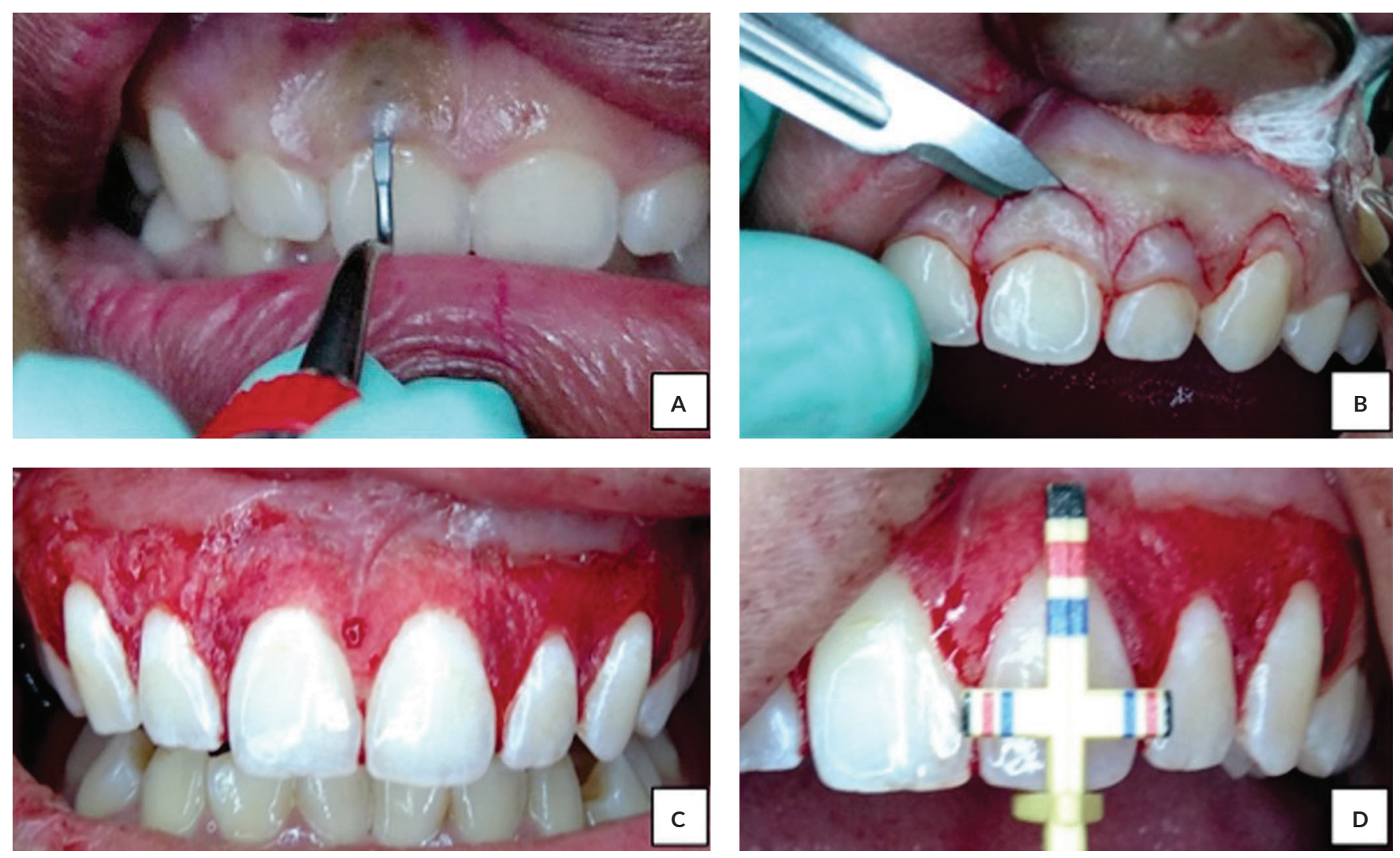

Figure 2. The sequence of the treatment. (A) Initial bone sounding, (B) Initial discontinue incision, (C) Post gingivoplasty, (D) Measurement for proportional width-to-height ratio. 

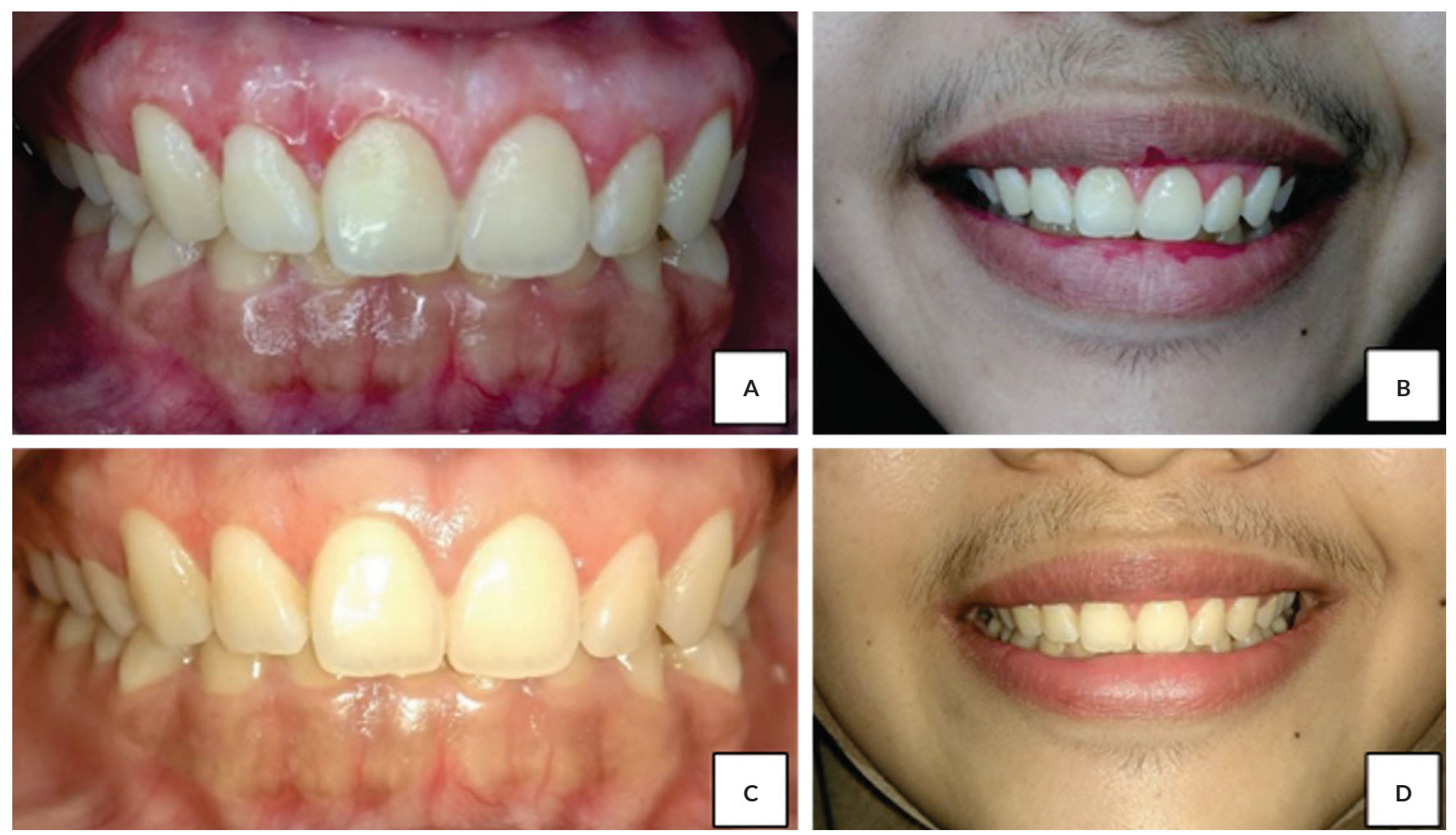

Figure 3. Result of the treatment. (A) Intraoral condition, (B) Smile profile one week after treatment, (C) Intraoral condition, and (D) smile profile one month after treatment.

Table 1. Clinical parameter measurement of the treatment

\begin{tabular}{|c|c|c|c|c|c|c|c|c|c|c|c|c|c|c|c|c|c|c|}
\hline \multirow{3}{*}{ Parameter } & \multicolumn{18}{|c|}{ Tooth } \\
\hline & \multicolumn{3}{|c|}{13} & \multicolumn{3}{|c|}{12} & \multicolumn{3}{|c|}{11} & \multicolumn{3}{|c|}{21} & \multicolumn{3}{|c|}{22} & \multicolumn{3}{|c|}{23} \\
\hline & D & $\mathbf{F}$ & $M$ & D & $\mathbf{F}$ & $M$ & D & $\mathbf{F}$ & $M$ & $M$ & $\mathbf{F}$ & D & $M$ & $\mathbf{F}$ & D & $M$ & $\mathbf{F}$ & D \\
\hline Probing Depth $(\mathrm{mm})$ & 2 & 1 & 2 & 2 & 2 & 2 & 2 & 2.5 & 2 & 1.5 & 2 & 1.5 & 2 & 2 & 2 & 2 & 1 & 2 \\
\hline Keratinized Gingiva (mm) & 8 & 6 & 8 & 8 & 8 & 8 & 9 & 8 & 9 & 8 & 8 & 8 & 8 & 8 & 8 & 8 & 7 & 8 \\
\hline Bone Sounding (BS) (mm) & 5 & 4 & 5 & 5 & 5 & 5 & 6 & 6 & 6 & 6 & 6 & 6 & 5 & 5 & 5 & 4 & 4 & 4 \\
\hline Crown width (W) (mm) & \multicolumn{3}{|c|}{8} & \multicolumn{3}{|c|}{7} & \multicolumn{3}{|c|}{8.5} & \multicolumn{3}{|c|}{8.5} & \multicolumn{3}{|c|}{7} & \multicolumn{3}{|c|}{8} \\
\hline Clinical Crown Height $(\mathrm{H})(\mathrm{mm})$ & \multicolumn{3}{|c|}{8} & \multicolumn{3}{|c|}{6} & \multicolumn{3}{|c|}{7} & \multicolumn{3}{|c|}{8} & \multicolumn{3}{|c|}{6} & \multicolumn{3}{|c|}{9} \\
\hline $\begin{array}{l}\text { Post treatment Clinical Crown } \\
\text { Height }\left(\mathrm{H}^{\prime}\right)(\mathrm{mm})\end{array}$ & \multicolumn{3}{|c|}{9} & \multicolumn{3}{|c|}{9} & \multicolumn{3}{|c|}{11} & \multicolumn{3}{|c|}{11} & \multicolumn{3}{|c|}{9} & \multicolumn{3}{|c|}{10} \\
\hline W/H Ratio (\%) & \multicolumn{3}{|c|}{100.0} & \multicolumn{3}{|c|}{116.7} & \multicolumn{3}{|c|}{121.4} & \multicolumn{3}{|c|}{106.3} & \multicolumn{3}{|c|}{116.7} & \multicolumn{3}{|c|}{88.9} \\
\hline W/H' Ratio (\%) & \multicolumn{3}{|c|}{80.0} & \multicolumn{3}{|c|}{77.8} & & 77.3 & & & 77.3 & & & 77.8 & & & 80.0 & \\
\hline
\end{tabular}

Note: D: Distal, F: Facial, M: Mesial

junction (CEJ), the mucogingival junction is apical to the crest of bone, and there is a wider gingival dimension than generally accepted as the mean, as given by Bowers ${ }^{7}$ and Aniamo and Löe. ${ }^{8}$ Subtype A means that the alveolar crest is located 1.5 to $2 \mathrm{~mm}$ from the CEJ. Another type is type II and subtypes B. Type II means the gingival dimension is normal. The free gingival margin is incisal or occlusal to the CEJ, and the mucogingival junction is positioned at the CEJ whereas subtype $\mathrm{B}$ means the alveolar crest is coincident with the CEJ. ${ }^{9}$
Preoperative surgical treatment planning is mandatory to ensure the intended treatment outcomes are achieved, namely the aesthetic proportion of the crown and healthy periodontal tissues. ${ }^{10}$ The amount of keratinized tissue determines what incision should be performed in planning treatment of altered passive eruption. If there is a good amount of tissue, an internal bevel can be easily used. On the other hand, if minimal keratinized tissue is observed, an intrasulcular incision is generally performed, associated with an apically positioned flap. Although there is no 
minimum amount of tissues considered as adequate for establishing health, the keratinized tissue must be considered as a noble structure related to aesthetics and the stabilization of the gingival sulcus. ${ }^{9,11,12}$

Treatment of choice for altered passive eruption class I subtype A was an aesthetic crown lengthening, which is characterized by sufficient gingival tissue coronal to the alveolar crest, allowing the surgical alteration of the gingival margin levels without the need for osseous recontouring. A gingivectomy or gingivoplasty procedure will usually suffice to establish the desired gingival margin position while simultaneously avoiding a violation of the biologic width. ${ }^{12-14}$

The distance between the CEJ and bone crest determines the need or otherwise for osteotomies to establish the space for the adaptation of the periodontal structures of the biological width. If a gingivectomy procedure is used to remove excess gingiva, but the new gingival margin position is too close to the underlying bone, the biologic width will be violated, and the gingival margin will usually rebound toward its original position. If the new gingival margin position is close to the underlying bone, a flap should be reflected, and an adequate amount of osteoplasty and ostectomy should be performed to re-establish an adequate biologic width. ${ }^{11}$ Thus, bone sounding must be performed before considering the surgical procedure technique. In this case, the bone sounding found that the margin of the gingiva to the alveolar crest was about 5-6 $\mathrm{mm}$, thus, excising 2-3 $\mathrm{mm}$ of gingiva will not violate the biological width. ${ }^{13}$ In this case management, we found that the gingival margin to be in a stable position after one month of surgery. This is consistent with a study by Dominguez et al. ${ }^{15}$ that found that a stable gingival margin position at one to six months after surgery can be observed if the gingival margin position is maintained at $\geq 3 \mathrm{~mm}$ coronal to the alveolar crest after the crown lengthening procedure.

Some aspects must be determined as a surgical evaluation for aesthetic symmetry. In evaluating the anterior segment for surgery, symmetrical analysis of gingival line, interpapillary line, individual gingival heights, posterior segments both individually and to each other, and also commission line must not be missed. To avoid aesthetic compromises, it is imperative that the interproximal tissues must be retained entirely, and that the surgery must be performed on the facial aspects of the teeth only. ${ }^{9,16}$

\section{CONCLUSION}

Aesthetic crown lengthening should be considered as a surgical component of aesthetic therapy to improve smile profile and eliminate gummy smile. Proper diagnosis of the smile type, hard and soft tissues relation is crucial for a successful treatment.

\section{Acknowledgment}

The author thanks the patients for giving consent for publication of images and other clinical information and also the Faculty of Dental Medicine Universitas Airlangga for support in publication of this case.

\section{Statement of Authorship}

Both authors participated in the data collection and analyses and approved the final version submitted.

\section{Author Disclosure}

Both authors declared no conflicts of interest.

\section{Funding Source}

This paper was funded by the Universitas Airlanggga.

\section{REFERENCES}

1. Pedraza JAQ, Salinas BRG, Fierro NC, Najera RIS, Trevino JJF, Delgado IR. Crown lengthening procedure using aesthetic measurement gauges: a case report. Int J Appl Dent Sci. 2017; 3(2): 95-7.

2. Lai JY, Silvestri L, Girard B. Anterior esthetic crown-lengthening surgery: A case report. J Can Dent Assoc. 2001; 67(10):600-3.

3. Moura D, Lima E, Lins R, Souza R, Martins A, Gurgel B. The treatment of gummy smile: integrative review of literature. Rev Clin Periodoncia, Implantol y Rehabil Oral. 2017;1 0(1):26-8.

4. Duruel O, Erduran NE, Tözüm TF. A modification for treatment of excessive gingival display: tooth-based lip-repositioning technique. Int J Periodontics Restorative Dent. 2020; 40(3):457-61.

5. KV M, Shahabe SA, Patil SG, Bijle MNA, Bhandi S. Esthetic crown lengthening: theoretical concepts and clinical procedures. Int J Contemp Dent. 2013; 3(3):33-7.

6. Rossi R, Brunelli G, Piras V, Pilloni A. Altered passive eruption and familial trait: a preliminary investigation. Int J Dent. 2014; 2014:874092.

7. Bowers GM. A study of the width of attached gingiva. J Periodontol. 1963; 34(3):201-9.

8. Ainamo J, Löe H. Anatomical characteristics of gingiva. A clinical and microscopic study of the free and attached gingiva. J Periodontol. 1966; 37(1):5-13.

9. Cohen ES. Altered Passive Eruption: The Gummy Smile. In: Atlas of cosmetic and reconstructive periodontal surgery. 3rd ed. BC Decker Inc; 2007. p. 259-69.

10. Kalsi HJ, Bomfim DI, Hussain Z, Rodriguez JM, Darbar U. Crown lengthening surgery: An overview. Prim Dent J. 2020 Jan; 8(4):48-53.

11. Joly JC, Mesquita CPF, Carvalho SR. Flapless aesthetic crown lengthening: A new therapeutic approach. Rev Mex Periodontol. 2011; 2(3):103-8.

12. Lee EA. Aesthetic crown lengthening: classification, biologic rationale, and treatment planning considerations. Pr Proced Aesthet Dent. 2004; 16(10):769-8.

13. Gupta G, Gupta R, Gupta N, Gupta U. Crown lengthening procedures-a review article. IOSR J Dent Med Sci. 2015; 14(4):27-37.

14. Hempton TJ, Dominici JT. Contemporary crown-lengthening therapy: a review. J Am Dent Assoc. 2010;141(6):647-55.

15. Domínguez E, Pascual-La Rocca A, Valles C, Carrió N, Montagut L, Alemany AS, et al. Stability of the gingival margin after an aesthetic crown lengthening procedure in the anterior region by means of a replaced flap and buccal osseous surgery: a prospective study. Clin Oral Investig. 2020; 24(10):3633-40.

16. Assaf M. Esthetic crown lengthening for upper anterior teeth: indications and surgica techniques. Int J Dent Med Res. 2014; 1(3): 86-91. 